\title{
Vergleichende Zusammenstellung
}

der Früchte und des Samens von Loranthus retusus, Loranthas europaeus und Viscum album.

Von $\mathrm{S}$ ch n a a e, Prediger in Danzig.

Fig. I. Samen von Loranthus retusus.

a) Aeusserste Stelle der Basis, mit der die

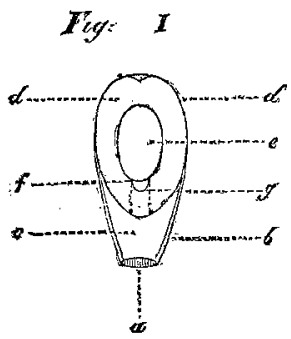
Frucht im Kelche der Pflanze befestiget ist.

b) Die Oberhaut der Beere.

c) Der Theil der Frucht, welcher (wie ich glaube) mit Viscin-Gefässen gefüllt ist.

d) Der dunkelbraun (im frischen Zustande wahrscheinlich roth) gefärbte Eiweisskörper.

e) Die Cotylen des Samens.

f) Die Stelle, an der die Cotylen verwachsen sind.

g) Der Caudex descendens der späteren Pllanze.

h) Wohrscheinliche Verlängerung des Caudex descendens, die aber bei dem Alter des vorliegenden Exemplares nicht deutlich zu erkennen war.

Fig. II. Samen von Loranthus europaeus.

Fig. II. a. Eiweisskörper mit seinem Embryo und seiner ihn umgebenden Haut im Durchschnitte.

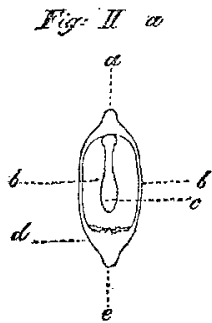

a) Convergenz der, den Eiweisskörper umgebenden weissen Haut an der Spitze des Samens.

b) Der Eiweisskörper.

c) Der Embryo.

d) Convergenz der, den Eiweisskörper umgebenden weissen Haut an der Basis des Samens.

e) Der Samenträger.

Fig. II. b. Der Eiweisskorper allein im Durcbschnitt (vergrössert).

EigF: II 6 .

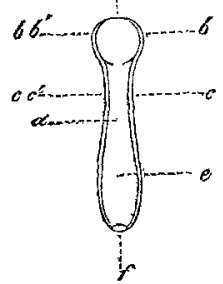

a) Das Kugelende.

$b$ u. $b^{\prime}$ ) Gränze der bis in das Kugelende hineinreichenden Bastbildung.

c) Die Bastbildung im Stammende.

d) Die Holzbildung im Embryo.

e) Der etwaige Cotyledonaltheil.

f) Offene Stelle des Embryo, durch welche derselbe die Nahrung in sich aufnimmt. 


\section{3}

Fig. III. Samen von Viscum album.

Fig. I.II $\boldsymbol{a}$. Verschiedene Form der Samen.

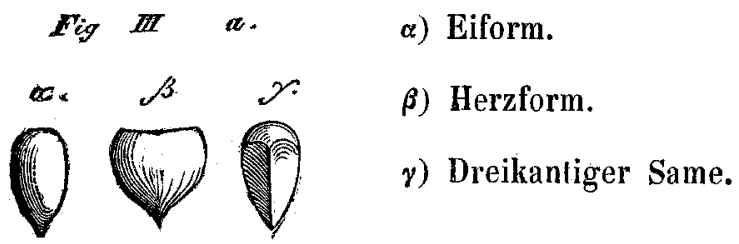

Fig. III. $b$. Samen mit einem Embryo im Flächendurchschnitt (vergrössert). Fig. III 8

a) Der Eiweisskörper.

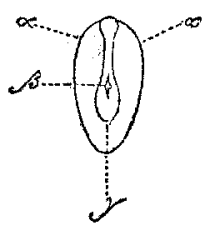

ß) Der Embryo.

y) Offene Stelle am Embryo, behufs dessen Ernährung.

Fig. III. c. Der Embryo allein (vergrössert).

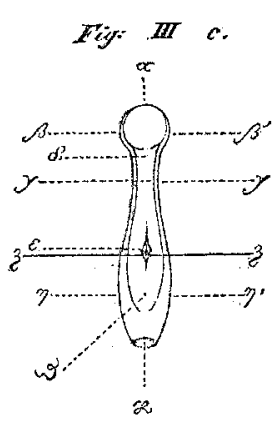

a) Das Kugelende.

$\beta$ u. $\beta^{\prime}$ ) Die in das Kugelende hineinreichenden ersten Bastformationen.

$\gamma \gamma$ ) Das Stammende.

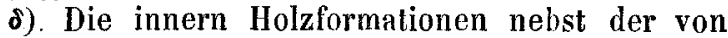
ihnen umschlossenen Medulla, die hier nicht bezeichnet sind.

ع) Dunkler Punct im Innern, bei dem der Knoten zur ersten Bastbildung zu suchen.

$\xi$ ६) Bezeichnung der Linie, in deren Richtung die Cotylen abirocknen.

$\eta \eta^{\prime}$ ) Die beiden Cotylen.

จ) Ernährungsorgan für die Holzformation und für die Medulla.

x) Offene Stelle, durch welche die Ernährung des im Eiweisskörper liegenden Embryos ausgeführt wird.

Vor einiger Zeit erhielt ich durch meinen lieben Freund, den sorgfältigen Pflanzenkenner und Pflanzenbeobachter Herrn Dr. Kli n smann in Danzig, eine bedeutende Zahl von Beeren des Loranthus europaeus, die er aus Wien erhalten hatte. Diese Mitheilung war mir um so interessanter, als ich Loranth. europ. noch nie gesehen hatte, wohl aber mich schon sechs Jahre lang mit Beobachtung des Viscum album beschäftigt hatte. Mein Interesse wurde noch erhöht, als ich ebenfalls durch Herrn Dr. Kl i n s ma n n noch einige Früchte von Loranth. retusus erhielt. Meine Bekanntschaft mit Visc. alb. kam mir bei der Untersuchung der, mir bis dahin unbekannten 
Früchten und Samen der beiden andern Lorantheen sehr zu statten. lch will hier eine vergleichende Darstellung von den Samen der genannten drei Pflanzen versuchen und an dieselbe auf Grund meiner Beobachtungen über das Anwurzeln des Visc. alb., auch meine Vermuthungen über das Anwurzeln des Loranthus europaeus und retusus kund geben. Mögen Solche, denen es vergönnt ist Loranth. europ., oder wohl gar Loranth. retusus sein Keimen und Anwurzeln zu beobachten, zusehen, ob ich aus den Analogien in der Structur der Samen dieser drei Pflanzen richtig auf ihr Anwurzeln geschlossen habe. Ich beginne mit dem, das ich am wenigsten kannte, mit dem Samen von Loranthus relusus. Die Samen, welche ich erhielt, waren im Jahre 1837 nach Europa gekommen; wann sie in Amerika gesammelt wurden, weiss ich nicht. Beim ersten Anblick derselben wunderte ich mich, dass ich nun Loranthus vor mir haben sollte. In diesem Zustande der Samen war jede Untersuchung unmöglich, die Samen lagen in einer Umbüllung, welche fast die Festigkeit und auch die Dicke der Schale einer Nuss von Corylus Avellana hatte. Ich wusste ja auch nicht, ob ich reife oder unreife Samen vor mir hatte; die grössten Samen mochten etwa einen haIben Zoll lang, und in ihrer grössten Ausdehnung etwa halb so dick als lang sein. Ich legte die Samen in Wasser und liess sie 48 Stunden in demselben liegen. Jetzt konnte ich durch die Section mehr erkennen. Ich werde nun den Theil der Frucht, mit welchem dieselbe an der Pflanze festsitzt ihre Basis, und das entgegengesetzte Ende ihre Spitze nennen. Von der Basis bis zu ihrer Spize war die Frucht mit einer schwarzen Haut überzogen. Späterhin üherzeugte ich mich, dass diese Oberhaut auch bei dieser Frucht wahrscheinlich wie bei andern farblos, aber durchscheinend ist, und dass sie mir schwarz erschien, weil das unter ihr Liegende eine sehr dunkle Färbung hatte. Von der Basis bis etwa auf ein Viertel der Fruchl war diese Oberhaut hier mit einer weisslichen Masse gefüllt, die ich auch jetzt noch, nach 17 Jăhren, in Fadenform durch Berührung mit dem Finger herausziehen konnte. Ich erkannte hierin ganz die Natur des Viscins bei Visc. alb., welches bei dieser Frucht dann sich zeigt, wenn man den Samen derselben aus der Haut der Beere sammt der Viscin-Kugel, die um den Samen liegt, herausdrückt, und dann die inneren Wände der Beerenhaut mit dem Finger berührt, wo sich dann auch das Viscin zu einem langen Faden ausspinnt. Ich halte dieses für die mit reinem Viscin gefüllten Gefässe der Mistelbeere, welche die Verlängerungen der äussern Bastlage des Viscum-Astes sind. Bei der Frucht von Pyrus Malus kann man recht genau sehen, welcher Theil der Frucht von der äussern und welcher von der innern Bastlage des Astes ausgeht und ich glaube, dass hierdurch die gewöhnliche Darstellung von der Bildung einer ersten and zweiten Membran, welche die sogenannte Nucleushöhle umschliessen, sich selbst verständlich erklärt. Doch ich habe den Gegenstand verlassen, von dem ich spreche. Ich halte die genannten weissen Fäden in der Frucht des Loranth. retusus für die Gefässe, a us denen und $\mathrm{d} u \mathrm{r} \mathrm{ch}$ die der im Innern liegende 
Same ernährt wird. Der übrige Theil der Oberhaut der Beere ist mit festeren Substanzen angefüllet. Zunächst liegt hier in Form einer Birne ein dunkelbrauner, etwas ins Röthliche spielender hohler Körper und sein Inneres ist ausgefüllt von dem eigentlichen Samen, der zwei kräftige Cotylen zeigt, die nach der Basis der Frucht hin verwachsen sind, wo man deutlich den Anfang zum Caudex descendens der nachherigen Pflanze erkennen kann.

(Fortsetzung folgt.)

\section{Beobachtungen}

über die örtliche Abstufung des Klimas und Einwirkung desselben auf die Pflanzen.

\section{Volt Johann Seywald.}

(Fortsetzung.)

Auf der Hälfte des Weges hört man schon von Ferne das Gebraus des sich über Felsen stürzenden Wassers, man befindet sich im Lueg, woselbst sich auf östlicher Seite unmittelbar aus dem Wasser eine hohe senkrechte Felsenwand erhebt. Das auf der westlichen Seite gelegene Gebirg ist zwar theilweise mit Bäumen bewachsen, wird aher im Frühjahre durch sich ablösende und auf die Strasse herabstürzende Felsentheile gefährlich. Etwas weiter erblickt man südlich den alle andern Gebirge weit überragenden Gippl, den Göller sieht man aber erst dann, wenn man bei der dortigen Schleife vorüber ist, er bildet den Hintergrund in südwestlicher Richtung des Thales.

Nach einer Viertelstunde erreicht man die schon erwähnte Eisen- und Stahlwaaren-Fabrik zu St. Aegydi und bald darauf den Markt St. Aegydi. *)

Unmittelbar ehe man zum Zerenn- und Walzwerk kommt, begegnet dem Beobachter jenseits der Unrecht-Traisen im Thale: Rhododendron hirsutum L., Silene alpestris J a cq., S. quadrifida L., Linaria alpina Mill., Rumex scutatus L., Primula auricula L., Alsine laricifolia L., Globularia cordifolia L., Campanula caespitosa S c op., Pinguicula alpina L., etwas höher Daphne Cneorum unter Erica carnea L. Auf minderhoch gelegenen Bergwiesen, und an Wegen der nächsten Uingebung: Gymnadenia conopsea R. Br. Gymnadenia odoratissima R ich., Hymantoglossum viride Rchb., Herminium Monorchis R. B r., Cypripedium Calceolus L., Ophris Myodes Jacq., Pyrola rotundifolia L., Majanthemum bifolium DC., Anthericum ramosum L., Primula officinalis J a c q., Gentiana cruciata L., Gentiana germanica W il1d., Valeriana tripteris L.. Digitalis grandiflora $\mathrm{L}$ a $\mathrm{m} \mathrm{k}$., Arnica montana L., Polygala chamaebuxus L., Tofieldia calyculata Wahlbg.,

*) In dem Aulsatze "Der Göller von St. Aegydi und seine Flora" you Freiherrn von Wi de $r$ s bach. (Botan. Wochenblatt, II. Jahrgang, Nr. 44) ist St. Aegydi irrig als ein Städtchen bezeichnet. 